\title{
EXISTENCE OF GENERAL BARGAINING SETS FOR COOPERATIVE GAMES WITHOUT SIDE PAYMENTS
}

\author{
BY LOUIS J. BILLERA
}

Communicated by David Blackwell, September 28, 1969

1. Introduction. The concept of a bargaining set for cooperative games with side payments was introduced by Aumann and Maschler in [2]. In [5] and [9] a particular bargaining set was defined which has the property that for each partition of the players, there is a payoff which is in this set. In [10], Peleg shows that although this bargaining set generalizes naturally to games without side payments, the existence theorem is no longer true.

In this paper we prove an existence theorem for a general class of bargaining sets for games without side payments. The treatment is similar to that of Peleg in [11], and the proofs rely directly on Peleg's results in [9]. It is hoped that the work here will provide a way of satisfactorily generalizing the classical bargaining set to the class of games without side payments. Several attempts at this will be mentioned.

For a survey of work in the no side payment theory, see [1]; for work on a related solution concept, the core, see [4] and [13].

The work presented here formed part of the author's doctoral dissertation in Mathematics for the Graduate Studies Division of the City University of New York. It was supported, in part, by the Army Research Office under contract number DA-31-124-ARO-D-366. The author would like to acknowledge the encouragement received from the late Professor Moses Richardson of C.U.N.Y. and Professor Michael Maschler of The Hebrew University of Jerusalem.

2. Definitions and main result. Let the set of players be $N$ $=\{1, \cdots, n\}$. For each $S \subset N$, let $E^{S}$ be the Euclidean space of dimension $|S|$ whose coordinates are indexed by the players in $S$. If $u \in E^{N}$ then $u^{S}$ will denote its projection onto $E^{S}$. If $x$ and $y$ are vectors we say $x \geqq y$ if $x \geqq y$ and $x \neq y$.

We use $\Omega_{S}$ and $\Omega_{S}^{+}$to denote respectively the nonnegative and the strictly positive orthant in $E^{S}$, i.e., $\Omega_{S}=\left\{x \in E^{S} \mid x \geqq 0\right\}$, and $\Omega_{S}{ }^{+}$ $=\left\{x \in E^{S} \mid x>0\right\}$.

For our purposes we will use the following definition of an $n$-person game with no side payments.

AMS Subject Classifications. Primary 9010; Secondary 9010, 5478.

Key Words and Phrases. Cooperative game, non-side-payment game, $n$-person game, bargaining sets, closed binary relations. 
DEFinition. An $n$-person game without side payments $\Gamma=\left\{V_{S}\right\}_{S \subset N}$ is a collection of sets satisfying the following conditions:

(1) For each $S \subset N, V_{S}$ is a nonempty, compact subset of $\Omega_{S}$.

(2) If $x \in V_{S}$ and $y \in \Omega_{S}$ is such that $y \leqq x$, then $y \in V_{S}$.

(3) For each $i \in N, V_{\{i\}}=\{0\}$.

For a discussion of these and other possible assumptions, and for an extensive bibliography, see Aumann [1]. Property 2 is called comprehensiveness.

Finally, we define for each $S \subset N$,

$\bar{V}_{S}=\left\{x \in V_{S} \mid\right.$ there is no $y \in V_{S}$ such that $\left.y>x\right\}$, and for each partition $B$ of $N$, we let

$$
X(B)=\underset{S \in \mathbb{B}}{\times} \bar{V}_{S}
$$

Let $B=\left\{S_{1}, \cdots, S_{m}\right\}$ be a partition of $N$, and for each $x \in X(B)$, let $R^{i}(x)$ be a binary relation on $S_{i}$, for $i=1, \cdots, m$. We define the bargaining set for the partition $\leftrightarrow$ and relations $R^{1}, \cdots, R^{m}$ (denoted $\left.M\left[\Theta ; R^{1}, \cdots, R^{m}\right]\right)$ to be the set of all $x \in X(B)$ such that whenever $i, j \in S_{k} \in \Re$, we have $i R^{k}(x) j$.

Letting, for $i \in S_{k} \in \Re$,

$$
E_{i}=\left\{x \in X(B) \mid i R^{k}(x) j \text { for all } j \in S_{k} \sim\{i\}\right\},
$$

we can now state our main result.

THEOREM 1. If the $R^{k}$ satisfy

(a) $E_{i}$ is closed in $X(B)$ for each $i \in N$,

(b) $E_{i} \supset\left\{x \in X(B) \mid x^{i}=0\right\}$ for each $i \in N$, and

(c) for each $x \in X(B)$, and each $S \in \Theta$, there is an $i \in S$ such that $x \in E_{i}$,

then $M\left[\Theta, R^{1}, \cdots, R^{m}\right] \neq \varnothing$.

3. Proof of Theorem 1. The proof of existence is a consequence of a series of lemmas which will be stated in this section. The idea of proof will be indicated for two of them. Complete proofs have been given in [3].

Definition. For $S \subset N, \bar{V}_{S}^{+}$is defined as follows:

(1) If $V_{S} \cap \Omega_{S}^{+} \neq \varnothing$, then $\bar{V}_{S}^{+}=\left\{x \in E^{S} \mid x \in \operatorname{closure}\left(V_{S} \cap \Omega_{S}^{+}\right)\right.$and there is no $y \in$ closure $\left(V_{S} \cap \Omega_{S}^{+}\right)$such that $\left.y>x\right\}$.

(2) If $V_{S} \cap \Omega_{S}^{+}=\varnothing$, then $\bar{V}_{S}^{+}=\left\{0^{S}\right\}$, where $0^{S}$ is the origin in $E^{S}$.

Lemma 1. $\bar{V}_{S}^{+} \subset \bar{V}_{S}$ for all $S \subset N$.

LEMma 2. If $V_{S} \cap \Omega_{S}^{+} \neq \varnothing$, then $\bar{V}_{S}^{+}=\left\{x \in E^{S} \mid x \in \operatorname{closure}\left(V_{S} \cap \Omega_{S}^{+}\right)\right.$ 
and there is no $y \in \operatorname{closure}\left(V_{S} \cap \Omega_{S}^{+}\right)$such that $y \geqq x$ and if $y^{i}=x^{i}$ then $\left.y^{i}=x^{i}=0\right\}$.

If we now define $X^{+}(\beta)$ as we defined $X(\beta)$, with $\bar{V}_{S}^{+}$in place of $\bar{V}_{S}$, it follows that $X(B)$ and $X^{+}(B)$ are closed sets, and $X^{+}(B) \subset X(B)$. It is clear that to prove Theorem 1 , it is enough to prove it in the case where $X^{+}(B)$ replaces $X(B)$ in all of $\$ 2$.

Definition. For each $S \subset N$, we define the set $H_{S}$ as follows. If $V_{S} \cap \Omega_{S}^{+} \neq \varnothing$, then

$$
H_{S}=\left\{x \in \Omega_{S} \mid \sum_{i \in S} x^{i}=1\right\}
$$

Otherwise

$$
H_{S}=\left\{x \in \Omega_{S} \mid \sum_{i \in S} x^{i}=0\right\}=\left\{0^{S}\right\}
$$

LEMma 3. For each $S \subset N$ there exists a continuous positive real valued function $d_{S}(h)$ defined for all $h \in H_{S}$ such that the function

$$
\phi_{S}: H_{S} \rightarrow \bar{V}_{S}^{+}
$$

defined for $h \in H_{S}$ by

$$
\phi_{S}(h)=d_{S}(h) h
$$

is a homeomorphism.

Proof. The case where $V_{S} \cap \Omega_{S}^{+}=\varnothing$ is trivial. If $V_{S} \cap \Omega^{+} \neq \varnothing$, we consider the map $\theta_{S}$ which projects $\bar{V}_{S}^{+}$onto $H_{S}$ along rays through the origin, and show it is a homeomorphism. That it is one-to-one follows from Lemma 2 , and that it is onto is a consequence of comprehensiveness. We define $\phi_{S}=\theta_{S}^{-1}$. It is clear that $\phi_{S}$ is of the required form, and that $d_{S}$ has the required properties.

The final lemma is a generalization of a result of Peleg [9], which is in turn a generalization of a well-known result of Knaster, Kuratowski and Mazurkiewicz [7] (see also [6, pp. 310-311]).

Lemma 4. Let $A_{1}, \cdots, A_{n}$ be closed subsets of $X^{+}(B)$. If for each $i \in N, A_{i} \supset\left\{x \in X^{+}(B) \mid x^{i}=0\right\}$, and for each $S \in B, \cup_{i \in S} A_{i}=X^{+}(B)$, then $\bigcap_{i \in N} A_{i} \neq \varnothing$.

Proof. Peleg has proven this lemma in the case where $X^{+}(B)$ is replaced by $H(B)=\chi_{S \in B} H_{S}$. (See $[9$, Corollary 2.5, p. 55].) By defining $\phi_{B}$ to be the product of the homeomorphisms $\phi_{S}$ and 
noting that $\phi_{S}$ takes $\left\{h \in H(B) \mid h^{i}=0\right\}$ homeomorphically onto $\left\{x \in X^{+}(B) \mid x^{i}=0\right\}$, the lemma follows immediately.

The proof of Theorem 1 is completed by observing that the sets $E_{\text {; }}$ satisfy the hypotheses of Lemma 4 and $M\left[\Theta, R^{1}, \cdots, R^{M}\right]=\bigcap_{i \in N} E_{i}$.

4. Applications. In this section, various attempts (none completely successful) to use Theorem 1 to generalize the classical bargaining set to games without side payments will be discussed.

Define the binary relation $i>(x) j$ to mean " $i$ has a justified objection against $j "$ as in $\left[10\right.$, p. 198]. Now let each of the relations $R^{k}(x)$ be the negation of $>(x)$. Then the bargaining set so defined is the one treated by Peleg in [10], and, as we noted earlier, the existence theorem is false for this set. In terms of Theorem 1, hypothesis (c) fails in general for this case. However, in games of pairs (see [10]), the theorem does apply, and we get a mild generalization of $[10$, Theorem 2.4, p. 199] (where convexity of each $V_{S}$ was assumed).

Now consider, at each $x$, the directed graph $G(x)$ of the relation $>(x)$. We may define $i R^{k}(x) j$ to mean whenever there is a directed path in $G(x)$ from $i$ to $j$, then there is also a directed path from $j$ to $i$. The bargaining set so defined can be shown to be equivalent to the classical bargaining set in games with side payments. However existence is an open question. Theorem 1 does not apply here since hypothesis (a) is not satisfied. We remark that a point $x_{0}$ in this bargaining set is characterized by $G\left(x_{0}\right)$ having the property that each of its connected components is strongly connected (i.e. between any two vertices $i$ and $j$ there is a directed path from $i$ to $j$ ).

The previous definition can be changed in such a way as to satisfy hypothesis (a). Define $i \gg(x) j$ if $x \in \operatorname{closure}(\{x \mid i>(x) j\})$. Let $H(x)$ be the graph of $\gg(x)$. Define $i R^{k}(x) j$ to mean whenever there is an $\operatorname{arc}$ from $i$ to $j$ in $G(x)$ then there is a directed path from $j$ to $i$ in $H(x)$. Theorem 1 can be applied here to guarantee existence. However the bargaining set so defined does not necessarily agree with the classical bargaining set for games with side payments, as the following example shows.

EXAMPLE. Let $n=7$. Suppose $v(1234)=100, v(15)=26, v(1256)=51$, $v(367)=26$, and $v(475)=26$. Let $v(S)=0$ for all other $S \subset N$. Let $B$ $=\{1234,5,6,7\}$. It can be shown that the payoff $(25,25,25,25,0,0,0)$ $\in X(B)$ does not belong to the classical bargaining set, however it does belong to the bargaining set defined above.

Finally, Nechemia [9] and Peleg [12] have given definitions of bargaining sets which use Theorem 1 to guarantee existence. Nechemia's set reduces to the classical set for side payment games, but he 
requires $B=\{N\}$ and the convexity of each $V_{S}$ for existence. Peleg's definition, on the other hand, has general existence properties, while it does not reduce in the classical case.

\section{BIBLIOGRAPHY}

1. R. J. Aumann, "A survey of cooperative games without side payments" in Essays in mathematical economics, M. Shubik (Editor), Princeton Univ. Press, Princeton, N. J., 1967, pp. 3-27. MR 35 \#1357; MR 34 \#5506.

2. R. J. Aumann and M. Maschler, The bargaining set for cooperative games, Advances in Game Theory, Princeton Univ. Press, Princeton, N. J., 1964, pp. 443-476. MR 31 \#1114.

3. L. J. Billera, On cores and bargaining sets for $n$-person cooperative games without side payments, Ph.D. Dissertation, The City University of New York, 1968.

4. - Some theorems on the core of an n-person game without side payments, SIAM J. Appl. Math. 18 (1970).

5. M. Davis and M. Maschler, "Existence of stable payoff configurations for cooperative games" in Essays in mathematical economics, M. Shubik (Editor), Princeton Univ. Press, Princeton, N. J., 1967, cf. Bull. Amer. Math. Soc. 69 (1963), 106108. MR 26 \#2332; MR 34 \#5506.

6. B. Knaster, K. Kuratowski and S. Mazurkiewicz, Ein Beweis des Fixpunktsatzes für n-dimensionale Simplexe, Fund. Math. 14 (1929), 132-137.

7. K. Kuratowski, Topologie. Vol. I, PWN, Warsaw, 1948; English transl., rev. aug. ed., Academic Press, New York, 1966. MR 10, 389; MR 36 \#840.

8. Asher Nechemia, Personal Communication, December, 1968.

9. B. Peleg, "Existence theorem for the bargaining set $M_{1}^{(i) "}$ in Essays in mathematical economics, M. Shubik (Editor), Princeton Univ. Press, Princeton, N.J., 1967, cf. Bull. Amer. Math. Soc. 69 (1963), 109-110. MR 26 \#2333; MR 34 \#5506.

10. - Bargaining sets of cooperative games without side payments, Israel J. Math. 1 (1963), 197-200. MR 30 \#2944.

11. - Equilibrium points for open acyclic relations, Canad. J. Math. 19 (1967), 366-369. MR 34 \#8825.

12. - The extended bargaining set for cooperative games without side payments, Research Program in Game Theory and Mathematical Economics, Department of Mathematics, The Hebrew University of Jerusalem, Israel, Research Memorandum No. 44, March 1969.

13. H. Scarf, The core of an N-person game, Econmetrica 35 (1967), 50-69. MR $38 \# 3051$.

Cornell University, IthacA, New York 14850 